\title{
FIELD ANALYSIS OF A DIELECTRIC-LOADED RECTANGULAR WAVEGUIDE ACCELERATING STRUCTURE
}

\author{
Liling Xiao, Wei Gai and Xiang Sun \\ Argonne National Laboratory, Argonne, IL 60439, USA
}

\begin{abstract}
In this paper, we present a detailed analysis of the modes of a dielectric-loaded rectangular waveguide accelerating structure. In general, the acceleration field in a synchronous acceleration mode is non-uniform in the two transverse dimensions. However, we could use an array of these structures rotated alternatively by 90 degrees to get a focusing-defocusing force continuously as a simple FODO lattice, while maintaining uniform energy gain. The expressions of characteristic parameters such as $\mathrm{R} / \mathrm{Q}$, group velocity and attenuation constant are given. The longitudinal wake field experienced by a relativistic charged particle beam in the structure is also presented. These analytical results are also compared with numerical calculations using the MAFIA code suite demonstrating the validity of our analytic approach.
\end{abstract}

\section{INTRODUCTION}

The possible applications of millimeter-wave dielectric waveguide accelerating structures to high energy accelerator have received much attention [1-6]. This class of accelerating structures can produce a high accelerating gradient and can easily be fabricated. Any dielectric slow wave structure can support travellingwave luminal accelerating modes. In order to avoid breakdown of the structure at higher accelerating fields, it is desirable to operate accelerating structures at higher frequencies [7], such as at W-Band. However, while higher frequency cylindrical structures would yield higher accelerating fields and shunt impedances, but the available energy per unit length for beam acceleration is limited. Therefore, simply scaling cylindrical geometry structures to higher frequencies may not lead to a higher luminosity accelerator. Tuning and fabricating small cylindrical structure is also becoming difficulty. It was pointed out by [4] that transverse wakefield and space charge force would be smaller than the axis symmetric structures.

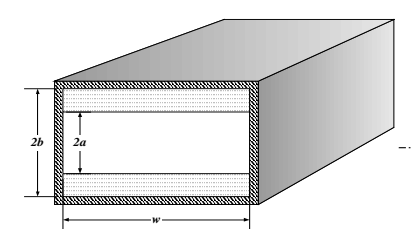

(a)

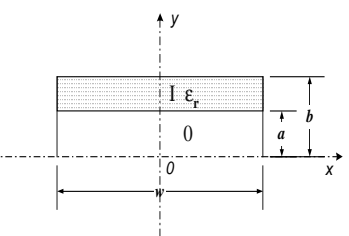

(b)
Fig.1. Dielectric-loaded rectangular guide (a), the half cross section (b)
In this paper, we study a slab type dielectric structure as shown in Fig.1 using a normal mode analysis. The electromagnteic properties of a planar dielectric lined waveguide had been analysed for an idealised case $(w \rightarrow \infty)$ in [4] and [5]. Wakefields were calculated in [4]. In this paper we calculate 3-D field distributions for a rectangular partially filled dielectric waveguide, and we give an exact solution for all the field components in terms of LSM (longitudinal section magnetic) and LSE (longitudinal section electric) modes using a mode matching method. This analysis provides a basis for studying the properties of this structure as an accelerator. We also present a wakefield calculation using the field analysis results.

\section{FIELD COMPONENTS}

The normal modes in dielectric-loaded guides are LSM and LSE modes [8] that have no $H$ or $E$ components normal to the dielectric interface. This corresponds to assuming the transverse direction to the interface normal vector to be the direction of propagation.

The general structure considered here is limited to the case of two $H$-plane slabs placed symmetrically. We also only analyse these modes with the longitudinal electric components at the central point since any other modes will not couple to the beam. It implies that the central plane in the $y-z$ view is a magnetic wall, so that only a half section with two subregions is needed for the analysis (shown in Fig.1(b)). In each region, the fields for LSM/LSE modes derive from the electric/magnetic Hertzian potential and satisfy the boundary conditions at the interface between dielectric and vacuum.

Due to the limited space, only the results for $\mathrm{LSM}_{\mathrm{mn}}$ modes are given here. The electric-type Hertzian potential function $\bullet_{\mathrm{e}}$ is

$\psi_{e m n}=\left\{\begin{array}{c}A_{m n} \sin \frac{m \pi}{w}\left(x+\frac{w}{2}\right) \sin k_{y m n}^{(0)} y \cdot e^{-j \beta_{m \pi}}, \quad 0<y<a \\ B_{m n} \sin \frac{m \pi}{w}\left(x+\frac{w}{2}\right) \cos k_{y m n}^{(1)}(b-y) \cdot e^{-j \beta_{m \pi}}, \quad a<y<b\end{array}\right.$
$k_{y m n}^{(0)}=k^{2}-\left(\frac{m \pi}{w}\right)^{2}-\beta_{m n}^{2}, \quad k_{y m n}^{(1)}{ }^{2}=\varepsilon_{r} k^{2}-\left(\frac{m \pi}{w}\right)^{2}-\beta_{m n}^{2}$

where $k=2 \bullet f / c$ is the propagation constant in free space.

The field components for $\mathrm{LSM}_{\mathrm{mn}}$ modes derive from $\bullet_{\mathrm{e}}$ and are given by 


$$
\begin{aligned}
& E_{x m n}=\left\{\begin{array}{c}
A_{m n} \frac{m \pi}{w} k_{y m n}^{(0)} \cos \frac{m \pi}{w}\left(x+\frac{w}{2}\right) \cos k_{y m n}^{(0)} y, 0<y<a \\
B_{m n} \frac{m \pi}{w} k_{y m n}^{(1)} \cos \frac{m \pi}{w}\left(x+\frac{w}{2}\right) \sin k_{y m n}^{(1)}(b-y), a<y<b
\end{array}\right. \\
& E_{y m n}=\left\{\begin{array}{c}
A_{m n} k_{c m n}^{2} \sin \frac{m \pi}{w}\left(x+\frac{w}{2}\right) \sin k_{y m n}^{(0)} y, 0<y<a \\
B_{m n} k_{c m n}^{2} \sin \frac{m \pi}{w}\left(x+\frac{w}{2}\right) \cos k_{y m n}^{(1)}(b-y), a<y<b
\end{array}\right. \\
& E_{z m n}=\left\{\begin{array}{c}
A_{m n}\left(-j \beta_{m n}\right) k_{y m n}^{(0)} \sin \frac{m \pi}{w}\left(x+\frac{w}{2}\right) \cos k_{y m n}^{(0)} y, 0<y<a \\
B_{m n}\left(-j \beta_{m n}\right) k_{y m n}^{(1)} \sin \frac{m \pi}{w}\left(x+\frac{w}{2}\right) \sin k_{y m n}^{(1)}(b-y), a<y<b
\end{array}\right. \\
& H_{x m n}=\left\{\begin{array}{c}
-A_{m n} \omega \varepsilon_{0} \beta_{m n} \sin \frac{m \pi}{w}\left(x+\frac{w}{2}\right) \sin k_{y m n}^{(0)} y, 0<y<a \\
-B_{m n} \omega \varepsilon_{0} \varepsilon_{r} \beta_{m n} \sin \frac{m \pi}{w}\left(x+\frac{w}{2}\right) \cos k_{y m n}^{(1)}(b-y), a<y<b
\end{array}\right. \\
& H_{z m n}=\left\{\begin{array}{c}
A_{m n}\left(j \omega \varepsilon_{0}\right) \frac{m \pi}{w} \cos \frac{m \pi}{w}\left(x+\frac{w}{2}\right) \sin k_{y m n}^{(0)} y, 0<y<a \\
B_{m n}\left(j \omega \varepsilon_{0} \varepsilon_{r}\right) \frac{m \pi}{w} \cos \frac{m \pi}{w}\left(x+\frac{w}{2}\right) \cos k_{y m n}^{(1)}(b-y), a<y<b
\end{array}\right.
\end{aligned}
$$

where $k_{c m n}{ }^{2}=(m \bullet / w)^{2}+\bullet_{m n}^{2}$. Note that $\exp \left(-j \bullet_{m n} z\right)$ has been omitted in these expressions for simplicity.

At the interface between the region $O$ and $I$, the tangential electric and magnetic field components are continuous:

$$
\begin{aligned}
& A_{m n} k_{y m n}^{(0)} \cos k_{y m n}^{(0)} a=B_{m n} k_{y m n}^{(1)} \sin k_{y m n}^{(1)}(b-a), \\
& A_{m n} \sin k_{y m n}^{(0)} a=B_{m n} \varepsilon_{r} \cos k_{y m n}^{(1)}(b-a),
\end{aligned}
$$

Dividing (4a) by (4b) yields the dispersion relation,

$$
k_{y m n}^{(1)} \tan \left(k_{y m n}^{(1)}(b-a)\right)=\varepsilon_{r} k_{y m n}^{(0)} \cot \left(k_{y m n}^{(0)} a\right)
$$

This is a transcendental equation of the general form of a complex function of $\beta_{m n}$ and $f$. For the inhomogeneous guide considered here, the dispersion relation must be solved numerically. An infinite number of discrete solutions exist.

From (4), the coefficient ratio A/B can be found, and, when substituted into (3), completes the solution.

\section{PROPERTIES OF THE LSM $_{11}$ AS ACCELERATION MODE}

In general, the relative advantages of accelerating structures can be understood with reference to figures of merit such as the ratio of the peak surface electric field to the axial acceleration field $E_{S} / E_{0}$, the group velocity $v_{g}$, the attenuation constant $\bullet$, and $R / Q$ which measures the efficiency of acceleration in terms of the given stored energy, etc. The definitions of these parameters will be quantified here. In this section, only the results pertaining to the $\mathrm{LSM}_{11}$ mode are given, because the $\mathrm{LSM}_{11}$ mode is the lowest luminal mode in our considered structures. And also because its symmetrical field distribution in $\mathrm{x}$ and $\mathrm{y}$-direction.

\subsection{The ratio of surface field $E_{s}$ to accelerating field $E_{0}$}

$\left|\frac{E_{s}}{E_{0}}\right|=\left|\frac{E_{y}(0, b)}{E_{z}(0,0)}\right|=\left|\frac{\left(\frac{\pi}{w}\right)^{2}+\beta^{2}}{k_{y} \beta} \sin k_{y} a\right|=\frac{\left(\frac{\pi}{w}\right)^{2}+\beta^{2}}{\frac{\pi}{w} \beta}\left|\sin \left(\frac{\pi}{w} a\right)\right|$

It is desirable to have this ratio as small as possible since the surface field contributes nothing to accelerating the beam but is responsible for breakdown of the structure.

\subsection{The group velocity $v_{g}$}

$v_{g}=\frac{P}{U} \quad P=\frac{1}{2} \iint E_{y} H_{x} d x d y$

$U=\frac{1}{2 L} \iiint\left(\varepsilon_{0} \varepsilon_{r}(y)\left(E_{x}^{2}+E_{y}^{2}+E_{z}^{2}\right)+\mu_{0}\left(H_{x}^{2}+H_{z}^{2}\right)\right) d x d y d z$

where $P$ is the power flow over the half section under consideration and $U$ is the stored energy per unit length in the half structure.

\subsection{The quality factor $Q$ (ignoring dielectric losses)} and the attenuation constant •

$$
\begin{aligned}
& Q=\frac{\omega U}{P_{\text {loss }}} \\
& P_{\text {loss }}=R_{s} \iint|\vec{H}|^{2} d s
\end{aligned}
$$

$$
R_{s}=\frac{1}{\delta_{\text {skin }} \cdot \sigma} \quad \text { and } \quad \delta_{\text {skin }}=\sqrt{\frac{2}{\mu_{0} \sigma \omega}}
$$

where $\bullet$ is the conductivity of the metal wall.

$$
\alpha=\frac{\omega}{2 Q v_{g}}=\frac{P_{\text {loss }}}{2 P}
$$

\subsection{The normalised shunt impedance $R / Q$}

$\frac{R}{Q}=\frac{E_{0}^{2} v_{g}}{\omega P}$

where $E_{0}$ is the maximum accelerating field on the $\mathrm{z}$ axis.

\section{NUMERICAL RESULTS}

Figure 2 shows the dispersion curves of the lower $\mathrm{LSM}_{\mathrm{m} 1}$ and $\mathrm{LSE}_{\mathrm{m} 1}$ modes for an X-band structure. Because the $\mathrm{LSM}_{11}$ mode is the lowest luminal mode and its field distribution pattern, it will be chosen as the accelerating mode. Figure 3 gives the normalized longitudinal electric field distributions of the $L S M_{11}$ synchronous mode as a function of $x$ and $y$ using the field analysis method. 


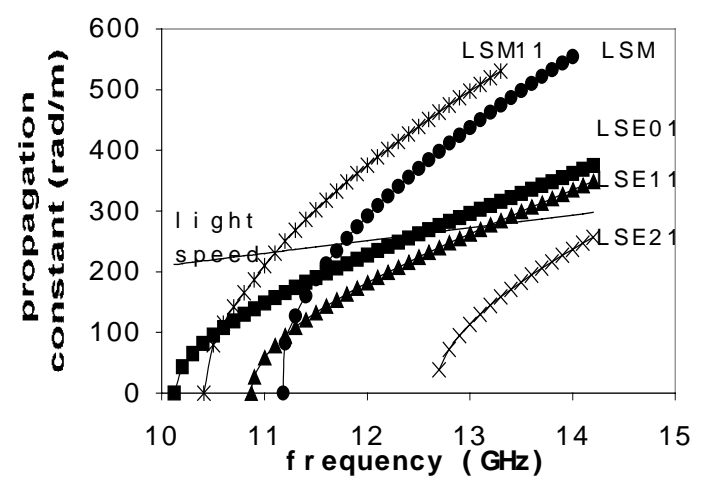

Fig.2. Dispersion curves of an X-Band dielectricloaded rectangular guide $(a=3 \mathrm{~mm}, b=5 \mathrm{~mm}$, $\mathrm{w}=\mathbf{2 3} \mathrm{mm}$ and $\bullet_{\mathrm{r}}=\mathbf{1 0}$ )

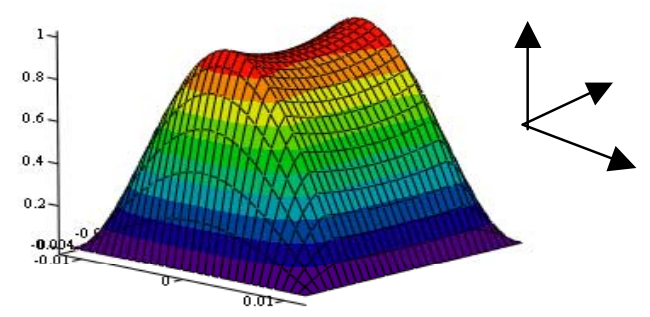

Ezfield

Fig.3 Normalised longitudinal electric field distributions at transverse section $(a=3 \mathrm{~mm}, b=5 \mathrm{~mm}$, $\mathrm{w}=\mathbf{2 3} \mathrm{mm}$ and $\bullet_{\mathrm{r}}=\mathbf{1 0}$ )

In vacuum, $k_{x}^{2}=-k_{y}{ }^{2}=(\bullet / w)^{2}, k_{y}$ is imaginary and of equal amplitude with $k_{x}$. This transverse dependence of the fields will result in focusing in the $\mathrm{x}$ direction and defocusing in y. Then, we could use an array of these structures rotated alternatively by 90 degrees to get a focusing-defocusing force continuously as a simple FODO lattice. While the integrated net acceleration force in the centre area will be very close to uniform, just as conventional iris loaded accelerating structure.

\section{WAKEFIELD ANALYSIS}

For a given structure, once R/Q is given, then one can obtain the longitudinal wakefield by simply using relation equation (11).

$$
\begin{aligned}
& W z(z)=\frac{1}{\sqrt{2 \pi} \cdot \sigma_{z}} \int_{-\infty}^{z} \sum_{i=1}^{\infty} E_{0 i} \cos \beta_{i}\left(z-z^{\prime}\right) \exp \left(-\frac{z^{\prime 2}}{2 \sigma_{z}^{2}}\right) d z^{\prime} \\
& E_{0 i}=\frac{q \omega_{i}}{4}\left(\frac{R}{Q}\right)_{i}
\end{aligned}
$$

We assume a Gaussian longitudinal beam shape (with bunch length $\bullet_{z}$ and charge $q$ ). The longitudinal wake $W_{z}(z)$ at distance $z$ behind the drive electron beam is given by [9]

In Fig.4, the longitudinal wake field obtained using equation (11) is shown. The dielectric-loaded rectangular waveguide operated at X-Band has the same parameters described in Section 4, and the beam shape is $\mathrm{q}=1 \mathrm{c}, \bullet_{\mathrm{z}}=2 \mathrm{~mm}$. We have compared the calculations with direct numerical integration of the Maxwell equations using the MAFIA[10] and shows good agreements.

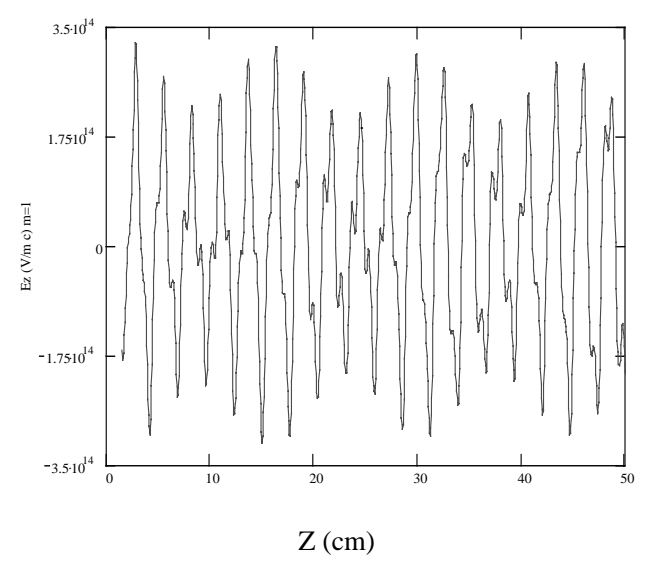

Fig. 4. Calculated longitudinal wake field in an XBand structure using the field analysis method in Section 2

In summary, analytical expressions for all the field components in a rectangular dielectric waveguide were given. It provides a basis for full accelerating field analysis and also can be used for wakefield calculations.

This work is supported by Department of Energy, High Energy Physics Division, Advanced Technology Branch under Contract No. W-31-109-ENG-38.

\section{REFERENCE}

[1] M. Rosing and W. Gai, Phys. Rev. D 42, 1829 (1990).

[2] King-Yuen Ng, Phys. Rev. D 42, 1819 (1990).

[3] S. Y. Park et al., Phys. Rev. E 62,.1266 (2000)

[4] A. Tremaine et al., Phys. Rev. E 56, 7204 (1997)

[5] Marc E. Hill, SLAC-R-560, (2000)

[6] P. Zhou et al., Rev. Sci. Instrum. 71, 2301 (2000)

[7] J.W.Wang and G.A.Loew, SLAC-PUB-7684, (1997)

[8] R. E. Collin, "Field Theory of Guided Waves", 2nd ed (Oxford University Press, 1991)

[9] H.H.Braun et al., CERN-CLIC-Note 364, 1998

[10] MAFIA, Version 4.0, Gesellschaft fur Computer-

Simulationstrechnik, Lauteshlagerstrabe 38, D-64289,

Darmstadt, Germany. 